\title{
OVERVIEW OF THE DEVELOPMENT OF ARTIFICIAL INTELLIGENCE TECHNOLOGY
}

\author{
Wei Hongyuan ${ }^{1}$, Deng Mingxing ${ }^{2}$ \\ ${ }^{I}$ Shanghai University of Engineering Science School of Mechanical Engineering, Shanghai, China \\ ${ }^{2}$ Shanghai University of Engineering Science School of Mechanical Engineering, Shanghai, China
}

\begin{abstract}
Abstract in recent years, with the development of mobile Internet, big data and the Internet of things, as well as the continuous breakthrough of large-scale computing capacity based on cloud platform, artificial intelligence technology develops rapidly and can independently complete the increasingly complex tasks completed by human beings through deep learning. The application of artificial intelligence technology is increasingly extensive. This paper summarizes the development status of artificial intelligence, expounds the meaning of artificial intelligence, sorts out the four modules of artificial intelligence technology system, and discusses the main application fields of current artificial intelligence.
\end{abstract}

Keywords: - Artificial Intelligence; Deep Learning; Neural Network; Machine Learning; The Image Processing ****

\section{INTRODUCTION}

Since the invention of "artificial intelligence" at Dartmouth in 1956, the meaning of artificial intelligence has been enriched and developed.Artificial Intelligence (AI) is a technology that USES machines to realize and replace human beings' functions of cognition, identification, analysis and decision-making. Its essence is to simulate the process of human consciousness and thinking information.Artificial intelligence integrates computer science, physiology, philosophy and other disciplines, and is a general term of computer simulation of human intelligence behavior science ${ }^{[1-3]}$.

The essence of artificial intelligence is to make the machine realize the response mechanism similar to human intelligence, and realize the improvement of production efficiency by virtue of the powerful computing ability of the machine.The concept of artificial intelligence can be divided into two parts for analysis."Manual" refers to a simple task that can be reached by labor without much thought."Intelligence" includes a series of difficult activities such as "consciousness" and "thinking".

\section{OVERVIEW OF THE DEVELOPMENT OF}

\section{ARTIFICIAL INTELLIGENCE}

The development of artificial intelligence has gone through three stages.The first stage was from 1950s to 1960 s, and the concept of artificial intelligence was put forward. The machine translation focusing on logical reasoning was mainly represented by knowledge expression such as propositional logic, predicate logic and heuristic search algorithm. The second stage was the 1970s and 1980s, and the expert system was proposed[4-5]. The algorithm research based on artificial neural network develops rapidly.
With the gradual improvement of semiconductor technology and computing hardware capability, artificial intelligence is gradually starting to break through, and distributed network reduces the computing cost of artificial intelligence. The third stage is that since the end of the 20th century, especially in 2006, the era of cognitive intelligence has entered the era of emphasizing data and independent learning. In 1997, the supercomputer "deep blue" beat the world champion in chess and caused a great stir in the world.In 2011, Watson, based on artificial intelligence technology, beat two human champions to win the award in the intelligence response program, making artificial intelligence technology once again the world's attention.In 2012, deep learning algorithm achieved a breakthrough in speech and visual recognition.In 2016, the emergence of "artificial go" has triggered thinking on how artificial intelligence will change human society[6-8].

\section{RESEARCH CONTENT OF ARTIFICIAL INTELLIGENCE}

Combined with the development and research of artificial intelligence technology, the artificial intelligence technology system can be summarized as four modules: machine learning, natural language processing, image recognition and human-computer interaction.as shown in table1.

Table 1: The main technical system of artificial intelligence

\begin{tabular}{|l|l|}
\hline designation & description \\
\hline $\begin{array}{l}\text { Machine } \\
\text { learning }\end{array}$ & $\begin{array}{l}\text { Supervised learning, unsupervised } \\
\text { learning, transfer learning, and intensive } \\
\text { learning }\end{array}$ \\
\hline $\begin{array}{l}\text { natural } \\
\text { language } \\
\text { processing }\end{array}$ & $\begin{array}{l}\text { Data sparseness and smoothness, Chinese } \\
\text { coding lexical analysis, syntactic analysis, } \\
\text { semantic analysis }\end{array}$ \\
\hline
\end{tabular}




\begin{tabular}{|l|l|}
\hline $\begin{array}{l}\text { picture } \\
\text { processing }\end{array}$ & $\begin{array}{l}\text { Feature extraction and selection, classifier } \\
\text { design, classification decision making, } \\
\text { neural network image recognition }\end{array}$ \\
\hline $\begin{array}{l}\text { man- } \\
\text { machine } \\
\text { interaction }\end{array}$ & $\begin{array}{l}\text { Interactive interface design, augmented } \\
\text { reality, geospatial tracking technology, } \\
\text { motion recognition technology }\end{array}$ \\
\hline
\end{tabular}

\subsection{Machine Learning}

Machine learning is a complicated subject with multiple technologies, and it is a science to solve how to achieve the cognitive ability of human thinking through algorithm technology[9].

The more professional definition of machine learning is that machine learning is a science that studies how computers do not actively learn without human intervention and can identify existing knowledge, especially with the function of improving algorithms to solve specific problems based on past experience.

Machine learning is a key technology of artificial intelligence, and algorithms play a major role in promoting the development of artificial intelligence.

At present, the mainstream multi-layer neural depth algorithm improves the ability to conclude data features from mass data and the ability to extract, describe and restore multi-layer features[10].

\subsection{Image Processing Technology}

Image processing technology enables the computer to have human visual functions, and can obtain, process and analyze and understand pictures or multidimensional data, including image acquisition, image filtering and adjustment, feature extraction, etc. The main research direction of computer vision is to analyze the collected photos or images through computer technology, to get deep data, and then to process them through mathematical methods, to get specific information of objects in photos or images. At present, the emergence of supercomputers and the development of cloud computing technology have provided strong support for the development of artificial intelligence. Computer vision technology has been widely used in face recognition, unmanned driving and other fields, adding color to people's lives.

\subsection{Natural Language Processing}

Natural language processing is a key subject in the field of artificial intelligence.

This discipline includes two aspects: natural language understanding and natural language generation. The former mainly explores whether the computer can understand the real meaning of natural language, while the latter mainly explores whether the computer can generate the natural language with specified meaning according to the needs of users. Therefore, natural language processing is a research subject combining computer science and linguistics. Natural language processing integrates linguistics, computer science, mathematics and other disciplines, and mainly studies computer systems that can realize natural language communication, including information retrieval, information extraction, speech tagging, syntactic analysis, speech recognition, grammar analysis, language translation and other technologies[11].

At present, natural language processing has been applied in all walks of life, greatly reducing the time and energy of manual recognition and understanding of text, and significantly improving the production efficiency.

\subsection{Man - Machine Interaction Technology}

Man-machine interaction technology refers to that computer system and users can communicate through the manmachine interaction interface, and the machine can provide users with a large number of prompts and request information through the output or display device. Users can enter relevant information and answer questions to the machine through the input device to achieve interaction. Human-computer interaction technology mainly includes computer graphics, interactive interface design, augmented reality and so on.

Man-machine interaction is one of the fastest developing technologies in user interface research. At present, many products and technologies have been developed, such as flexible display, 3D display, multi-touch touch screen technology, handwritten Chinese character recognition system, digital ink technology and implicit input technology based on sensor to capture users' intention.

\section{MAINAPPLICATION OF ARTIFICIAL INTELLIGENCE}

Artificial intelligence technology has developed rapidly in recent years and has been successfully applied in many industries. According to the characteristics of artificial intelligence, it is easier to replace the work content that is relatively clear in process and rules.

Artificial intelligence can be divided into special artificial intelligence and general artificial intelligence from its application range. At present, the application scenarios of artificial intelligence technology in various industries are gradually increasing. Data resources are the basic materials for machine learning and training. Through in-depth learning of data, the decision-making parameters are constantly optimized and gradually become as intelligent as human beings.

At present, with the rapid development of the Internet and the Internet of things, especially the big data, the data generated online is exploding and the abundance of materials for machine learning and training is greatly increased. In addition to the Internet industry, the data base of medical, financial, education, household, automobile, 
retail and other industries is relatively perfect, data resources are more abundant, its industry application is more extensive and its application is relatively mature[12].

\subsection{Medical Field}

application of artificial intelligence in medical field the application of artificial intelligence in medical field is mainly reflected in auxiliary diagnosis, rehabilitation intelligent equipment, medical records and medical image understanding, surgical robots and other aspects. The first is to identify medical images through machine vision technology, so as to help medical personnel shorten the reading time, improve work efficiency and reduce the rate of misdiagnosis.

Second, based on natural language processing, "understand" the description of the patient's symptoms, and then conduct content comparison and in-depth learning according to the disease database, so as to assist the diagnosis of the disease.

Some companies have begun experimenting with customizing treatment plans for patients based on massive amounts of data and machine learning. According to relevant data, the artificial intelligence system developed by harvard medical school has achieved 92 percent accuracy in identifying cancer cells in the images of breast cancer cases, and 99.5 percent accuracy in diagnosis combined with the analysis of artificial pathology. In addition, the machine learning algorithm can be used to build a variety of auxiliary diagnosis models for diseases, and the diagnosis opinions can be calculated by analyzing patients' data to identify diseases.

At present, combined with the analysis of medical experts, the auxiliary diagnostic models of artificial intelligence in the fields of tumor, cardiovascular, facial features and neurology are close to the level of doctors[13-15].

\subsection{Financial Field}

The application of artificial intelligence in the financial field mainly includes intelligent investment, investment decisionmaking, intelligent customer service, precision marketing, risk control, anti-fraud, intelligent claims settlement, etc. The most widely used investment advisory business, the industry called "smart investment customers."Famous intelligent investment platforms around the world include Wealthfront, Betterment, Personal Capital,etc. Robo-advisor is an innovative financial technology that has swept Wall Street in recent years.

In 2009, smart investment banking emerged in the United States, and by the end of 2015, a group of emerging financial technology companies began to expand the Chinese smart investment market.Through big data, intelligent investment clients can obtain personalized risk preferences and change rules of customers, customize personalized investment plans according to customers' risk preferences and combined with the algorithm model. At the same time, they can use the Internet to make real-time tracking and adjustment on customers' personalized asset allocation plans[16].

\subsection{Household Field}

the application of artificial intelligence in the household field with the development of artificial intelligence technology, smart home has entered the daily life of consumers, changing people's lifestyle. Although there are more and more sensors on the market, most of the smart home products rely on mobile phone control.

It can sense the surrounding environment well, and there are not many applications that truly reflect the intelligent scene. The intelligence of household products mainly lies in the ability to comprehensively analyze and judge the surrounding environment and satisfy users' emotional experience in home. With the development of artificial intelligence technology, artificial intelligence will bring more advanced induction methods, understand users' psychology, preferences, habits, etc., comprehensively perceive and sense the home environment through the interaction function of the induction system, calculate and execute corresponding instructions.

\section{CONCLUSION}

Artificial intelligence is a new subject, it is the interdisciplinary subject of cybernetics, information theory, computer science, mathematical logic, neurophysiology and so on[17].

With the development of artificial intelligence technology, the application scenarios will be enriched continuously, and the sustainable development of its supporting technologies will be driven, and the market scale of artificial intelligence will gradually expand.

The arrival of the AI era will be a new opportunity and challenge for individuals, enterprises and countries.

In the era of rapid development of artificial intelligence, many jobs will be replaced. Therefore, education and improvement of personal quality should be changed to meet the requirements of future development.

In the future, people will be liberated from bondage due to the arrival of the era of artificial intelligence, and they will be truly committed to the field suitable for their own development.

\section{REFERENCES}

[1] Zhangni, xuwenshang, wangwenwen. Overview of the development and application of artificial intelligence technology $[\mathrm{J}]$. Coal mine machinery, 2009, 30(02):4-7. 
[2] Tianjinping. Overview of the development of artificial intelligence $[\mathrm{J}]$. Science and technology square, 2007(01):230-232.

[3] Chen xuesong, Yang yimin. Overview of intensive learning research $[\mathrm{J}]$. Computer application research, 2010, 27(08):2834-2838+2844.

[4] Xiaoboda, zhouguofu. Overview of the development and application of artificial intelligence technology [J]. Fujian computer, 2008,34(01):98-99+103.

[5] Suruoqi. Overview of the development and application of artificial intelligence [J]. Electronic world, 2018(03):84+86.

[6] Xuziming, wangzhen, tianyangfeng. Overview of artificial intelligence [J]. Scientific and technological innovation, 2018(06):99-100.

[7] Suxuan. Overview of artificial intelligence [J]. Digital communication world, 2018(01):105+112.

[8] Zhaochenyang. Overview of machine learning [J]. Digital communication world, 2018(01):109+112.

[9] Lv Wei, Zhong Zhen yi, zhangwei. Artificial intelligence technology review $[\mathrm{J}]$. Journal of Shanghai electric technology, 2018, 11 (01): 62-64.

[10] Sun ye. Overview of the development of artificial intelligence $[\mathrm{J}]$. China new communications, 2008,20(06):52-53.

[11] Tangyuwei, liuyongfeng. Research review on the development of artificial intelligence in the field of news communication $[\mathrm{J}]$. Western radio and television, 2018(05):36-37.

[12] Wufei, Yang chunhua, LAN xuguang, ding jinliang, zhengnanning, GUI weihua, gao wen, chai tianyou, qianfeng, li deyi, pan yunhe, hanjunwei, fujun, liuke, song su and wuguozheng. Review and prospect of artificial intelligence $[\mathrm{J}]$. China science foundation, 2012, 32(03):243-250.

[13] Liyuhuan. Overview of artificial intelligence [J]. Science and technology innovation guide, 2016, 13(16):77-78.

[14] Liwei. Overview of machine learning [J]. Science and technology vision, 2017(12):149.

[15] Patrick g. Clark,ArvinAgah,Gerhard w. Cibis. Applied Artificial Intelligence Techniques for Identifying the Lazy Eye Vision Disorder[J]. Journal of Intelligent Systems, 2011,20(2).

[16] JacekGrudzie. The Development of Computer shaky Using Artificial Intelligence[J]. International Journal of Information and Communication Technologies in Education, 2014, 3(1).

[17] Micha transferred Klincewicz. Artificial Intelligence as a Means to Moral Enhancement[J]. Studies in Logic, Grammar and Rhetoric, 2016, 48(1). 